\title{
Carcinomes indifférenciés du nasopharynx à Dakar, une zone supposée non endémique : à propos de 13 cas
}

\author{
Undifferentiated Carcinoma of the Nasopharynx in Dakar, an Area Supposed \\ to be Non-Endemic: about 13 Cases
}

\author{
A.M. Gaye · F.G. Mouamba $\cdot$ M.J. Diemé $\cdot$ D. Niang $\cdot$ I. Thiam $\cdot$ C.M.M. Dial \\ Reçu le 10 février 2018; accepté le 1 juin 2018 \\ (C) Société de pathologie exotique et Lavoisier SAS 2018
}

Résumé Les auteurs étudient et discutent les aspects anatomopathologiques du carcinome indifférencié du nasopharynx (UCNT) au Sénégal à propos de 13 cas. Ils recherchent par ailleurs à faire le lien entre ce cancer et la présence d'une infection par le virus d'Epstein-Barr (EBV) chez ces patients. Il s'agit d'une série rétrospective de patients porteurs d'UCNT, diagnostiqués sur base histologique à Dakar sur 10 ans, et confirmés par l'immunohistochimie. Une recherche d'EBV a été effectuée sur ces cas en hybridation in situ par la sonde EBER. Quinze cas diagnostiqués sur base histologique ont pu bénéficier de l'immunohistochimie et 13 cas ont été confirmés, soit $87 \%$. L'âge moyen était de 28,37 ans avec des extrêmes de 5 et 56 ans. Le sexe masculin prédominait, avec un sex-ratio de $8 / 3$. Les lésions macroscopiques étaient de type ulcéro-bourgeonnant pour les cas se révélant par une masse du cavum. L'aspect histologique se traduisait par une prolifération de cellules indifférenciées atypiques, aux noyaux volumineux, clairs, fortement nucléolés, aux cytoplasmes éosinophiles, peu abondants. Le stroma était de type lymphoïde. L'hybridation in situ avec la sonde EBER montrait un marquage diffus modéré ou intense des cellules tumorales pour 10 cas, soit $77 \%$. Le nombre de cas notés dans notre étude, tenant compte de la période de 10 ans considérée, est révélateur du caractère rare de ce type de cancer à Dakar. Si à l'échelle mondiale, le virus d'EpsteinBarr est constamment associé à l'UCNT, pour certains de

A.M. Gaye · F.G. Mouamba $(\bowtie) \cdot$ I. Thiam Laboratoire d'anatomie et cytologie pathologiques du CNHU Aristide Le Dantec, Dakar, Sénégal e-mail : mfabiengael@yahoo.com

M.J. Diemé · D. Niang

Laboratoire d'anatomie et cytologie pathologiques

du CNHU de Fann, av. Cheikh Anta Diop, Dakar, Sénégal

C.M.M. Dial

Laboratoire d'anatomie et cytologie pathologiques

du CNHU de Grand Yoff, Dakar, Sénégal nos cas nous n'avons pas pu mettre en évidence EBV, suggérant l'implication possible d'autres facteurs de risque, tel que le papillomavirus humain (HPV).

Mots clés Carcinome indifférencié du nasopharynx · Virus d'Epstein-Barr · Papillomavirus humain · Hôpital · Dakar · Sénégal · Afrique intertropicale

Abstract Undifferentiated carcinoma of the nasopharynx (UCNT) has variable incidence throughout the world. Senegal is supposed to be a non-endemic zone. There is a constant connection with the Epstein-Barr virus. In this work, the authors study and discuss the pathological aspects of undifferentiated carcinoma of the nasopharynx in 13 cases. They also seek to make the link between this cancer and the presence of an Epstein-Barr virus infection in these patients. This is a retrospective series of cases involving patients with UCNT, diagnosed on histological basis in Dakar and confirmed by immunohistochemistry. An EBV search was performed on these cases through in situ hybridization by the EBER probe. 15 cases diagnosed on histological basis were able to benefit from immunohistochemistry, and 13 cases were confirmed, ie $87 \%$. The mean age was 28.37 years with extremes of 5 and 56 years. The male sex predominated, with a sex ratio of $8 / 3$. The macroscopic lesions were of ulcerous-budding type for cases revealed by a mass of the cavum. The histological aspect resulted in a proliferation of atypical undifferentiated cells, with large, clear, strongly nucleated nuclei, with eosinophilic cytoplasm, scarcely abundant. The stroma was of the lymphoid type. In situ hybridization with the EBER probe showed moderate or intense diffuse scarring of the tumor cells for 10 cases, ie $77 \%$. The number of cases noted in our study taking into account the period of 10 years considered, is indicative of the rare nature of this type of cancer in Dakar. While globally, Epstein-Barr Virus is consistently associated with UCNT, for some of our cases we have not been able to 
highlight EBV, suggesting the possible involvement of other risk factors, such as HPV.

Keywords Undifferentiated carcinoma of the nasopharynx • Epstein-Barr virus · Human papillomavirus · Hospital ·

Dakar $\cdot$ Senegal $\cdot$ Sub-Saharan Africa

\section{Introduction}

Le carcinome indifférencié du nasopharynx, dit UNCT (Undifferenciated Carcinoma of Nasopharyngeal) est, comme les cancers nasopharyngés en général, d'incidence variable à travers le monde, avec d'une part des zones à risque élevé telles que le Sud-Est asiatique, et à risque intermédiaire comme l'Afrique du Nord, et de l'autre, les zones à risque faible $[4,9]$. Le Sénégal n'est pas cité comme une zone de forte endémicité. Il s'agit du type histologique le plus fréquent des carcinomes nasopharyngés avec notamment plus de $90 \%$ des cas dans certains pays du Sud-Est asiatique, en Chine par exemple [11]. Le pic se situe vers 40-50 ans, avec une distribution souvent bimodale (20-30 ans et après 50 ans) en particulier dans les zones à faible risque. L'homme est plus fréquemment touché avec un sex-ratio de 2,5 à 3 [5]. La composante virale est essentielle dans son étiopathogénie, et il y a une liaison quasi constante avec le virus d'Epstein-Barr. Il est de diagnostic difficile à l'histologie, compte tenu de son caractère indifférencié, et pose fréquemment un problème de diagnostic différentiel avec certaines lésions sarcomateuses, lymphomateuses et bien d'autres. Mieux affiner son diagnostic peut s'avérer bénéfique pour le malade, car sa sensibilité thérapeutique et son pronostic ne sont pas identiques à ceux des autres types histologiques de carcinomes nasopharyngés ou même à ceux des lésions malignes non carcinomateuses.

Le but de notre travail était d'étudier au travers d'une courte série de 13 cas sénégalais, les aspects anatomopathologiques des UCNT et de faire le lien avec l'infection par le virus d'Epstein-Barr.

\section{Patients et méthodes}

Dans cette série rétrospective, il s'est agi de colliger tous les cas d'UNCT, diagnostiqués sur base histologique de janvier 2007 à décembre 2015, dans les laboratoires publics d'anatomie et cytologie pathologiques de Dakar. A noter que ces cas devraient être confirmés par l'immunohistochimie en utilisant les anticorps anti-pancytokératine AE1-AE3. Les cas diagnostiqués d'UCNT sur base histologique, mais non confirmés à l'immunohistochimie, et les cas pour lesquels l'immunohistochimie n'a pu être effectuée ont été exclus de notre étude. L'hybridation in situ par la sonde EBER, nous a permis de rechercher la présence ou non du virus d'Epstein-Barr. L'immunohistochimie et l'hybridation in situ ont été réalisées avec le concours du laboratoire d'anatomie et cytologie pathologiques du Centre hospitalier universitaire d'Amiens (France). Les blocs ont été acheminés en 2 séries. Une première série de 8 blocs et une autre de 5 , en pli fermé, respectivement par DHL et par un passager.

Par ailleurs, la présence d'EBV en hybridation in situ a été évaluée à travers un score de positivité EBER allant de 0 à 3 . Ainsi, ce score était coté 0 lorsque aucune cellule n'était marquée, était coté 1 lorsque le marquage nucléaire des cellules intéressait moins de $10 \%$ du volume tumoral, coté 2 lorsque le marquage nucléaire des cellules intéressait entre $10 \%$ et $49 \%$ du volume tumoral ; et enfin il était coté 3 lorsque le marquage nucléaire des cellules intéressait plus de $50 \%$ du volume tumoral.

\section{Résultats}

Quinze cas diagnostiqués sur base histologique ont pu bénéficier de l'immunohistochimie, et 13 cas ont été confirmés, soit $87 \%$, exprimant fortement la pancytokératine AE1/AE3 (Fig. 1). Les deux autres cas se sont avérés être finalement un rhabdomyosarcome alvéolaire et un lymphome. Pour ces 13 cas ayant exprimé AE1/AE3, l'immuno-marquage avec la protéine de latence LMP1 n'a pu être réalisé, cependant les marqueurs neuroendocrines ont été négatifs et donc ces cas ont été considérés comme des carcinomes indifférenciés.

L'âge moyen était de 28,37 ans avec des extrêmes de 5 et 56 ans. On a noté une prédominance du sexe masculin avec un rapport homme/femme de 8/3.

Sur le plan anatomopathologique, le prélèvement était une biopsie ganglionnaire pour 3 cas, une biopsie du cavum pour 8 cas et pour les 2 autres cas, il a intéressé à la fois le cavum et le ganglion. Ces adénopathies étaient de localisation cervicale, souvent bilatérale. Dans les cas où l'endoscopie avait objectivé une lésion du cavum, celle-ci était macroscopiquement de type ulcéro-bourgeonnant.

À l'histologie, les lésions se sont traduites par une prolifération de cellules indifférenciées irrégulières, disposées en nappes, parfois en massifs dyscohésifs. Leurs noyaux étaient de grande taille, aux contours irréguliers, avec la chromatine tantôt dense, tantôt en motte, et le nucléole visible voire proéminent. Leurs cytoplasmes étaient peu abondants, clairs, mais le plus souvent éosinophiles. Ces cellules étaient dissociées par un stroma de type lymphoïde. C'est sur cet aspect que le diagnostic d'UCNT était évoqué à l'histologie sur coloration HE (Fig. 2).

À l'hybridation, le Tableau 1 résume le marquage nucléaire des cellules tumorales avec la sonde EBER pour nos 13 cas, en tenant compte du score de positivité sus évoqué. 


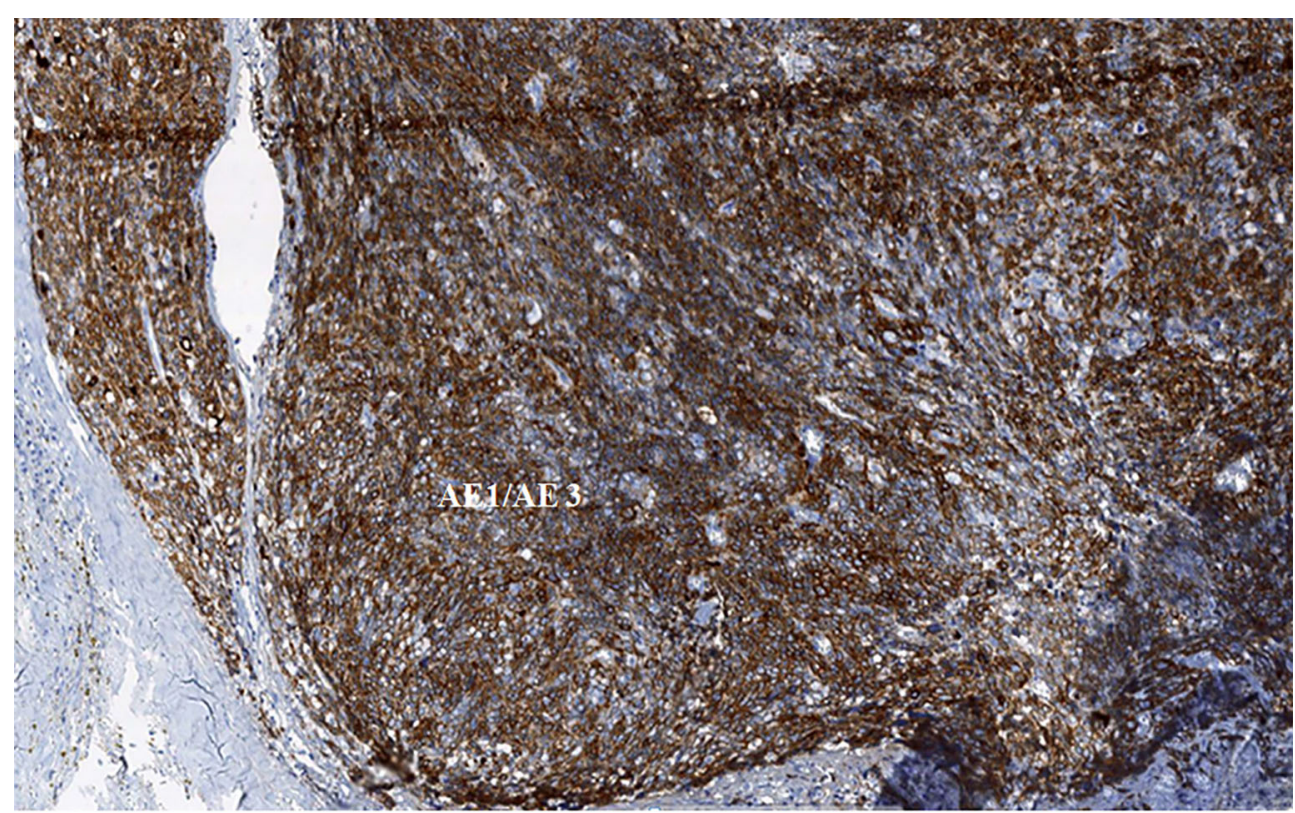

Fig 1 Immunomarquage des cellules tumorales avec la pancytokératine AE1/AE3 (x250)/ Immunolabelling of tumoral cells using pancytokeratin AE1/AE3 (x250)

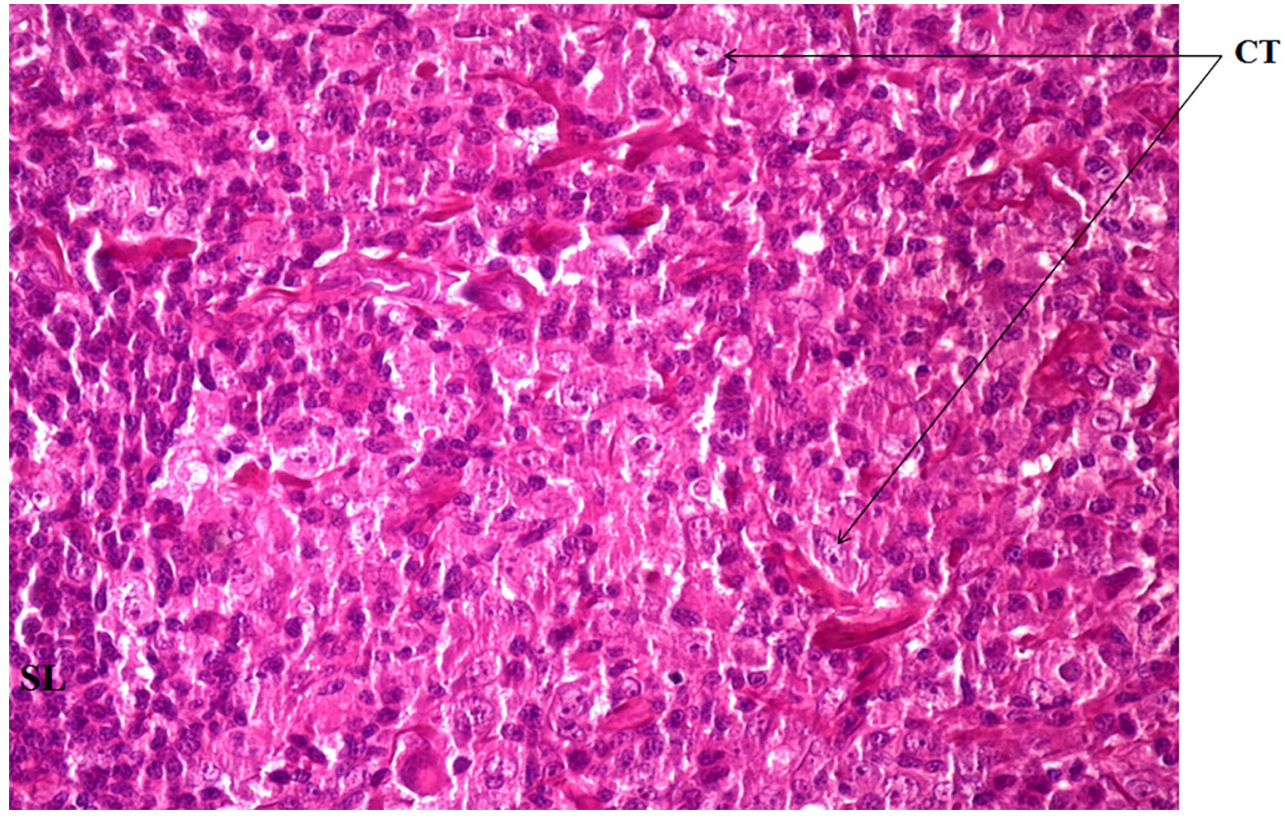

Fig 2 Aspect histologique d'UCNT (HEx400) / Histological aspect of UCNT (HEx400) Nappes de cellules tumorales (CT) indifférenciées, aux noyaux augmentés de volume, pales, souvent nucléolés. Leurs cytoplasmes sont éosinophiles, peu abondant. Le stroma est de type lymphoïde (SL)

Il y a eu un marquage nucléaire avec la sonde EBER pour 10 des 13 cas, soit une positivité EBER de $77 \%$. Le score de positivité EBER a été nul dans 3 cas, soit $23 \%$. Pour les 10 cas positifs, on a noté 9 cas avec un score de positivité EBER coté 3 , correspondant à un marquage nucléaire diffus et intense des cellules tumorales et 1 cas avec un score de positivité EBER de 2, soit un marquage intense mais focal des cellules tumorales infectées par EBV. Les différents marquages nucléaires des cellules tumorales avec la sonde EBER sont illustrés par la Figure 3. 


\section{Discussion}

Le carcinome indifférencié du nasopharynx est une des entités histologiques des cancers du nasopharynx. Ces cancers du nasopharynx sont surtout des carcinomes (communément appelés NPC), qui constituent 75 à $95 \%$ des cancers du nasopharynx dans les populations à faible risque et pratiquement tous les cancers du nasopharynx dans les populations à haut risque [11]. Il y a eu plusieurs évolutions quant à leur classification histologique au niveau de l'OMS, dans le but certainement de mieux cerner les frontières entre les différentes entités histologiques, aux facteurs étiologiques pas toujours communs et au pronostic aussi différent. En effet, la classification qui est d'actualité est celle de l'OMS 2005, qui distingue deux principaux types, celui des carcinomes kératinisant (ex type I) et celui des carcinomes non kératinisant, et un troisième ajouté qui concerne les carcinomes épidermoïdes basaloïdes [3]. La catégorie des carcinomes non

Tableau 1 Score de positivité du marquage des cellules tumorales par la sonde EBER en HIS (Laboratoire d'anatomie et cytologie pathologiques du CHU d'AMIENS) / Positivity score for the marking of tumoral cells using EBER ISH probe

\begin{tabular}{|lllll|}
\hline $\begin{array}{l}\text { Score de positivité } \\
\text { EBER }\end{array}$ & Score 0 & Score 1 & Score 2 & $\begin{array}{l}\text { Score } \\
\mathbf{3}\end{array}$ \\
\hline Nombre de cas & 03 & 0 & 01 & 09 \\
\hline
\end{tabular}

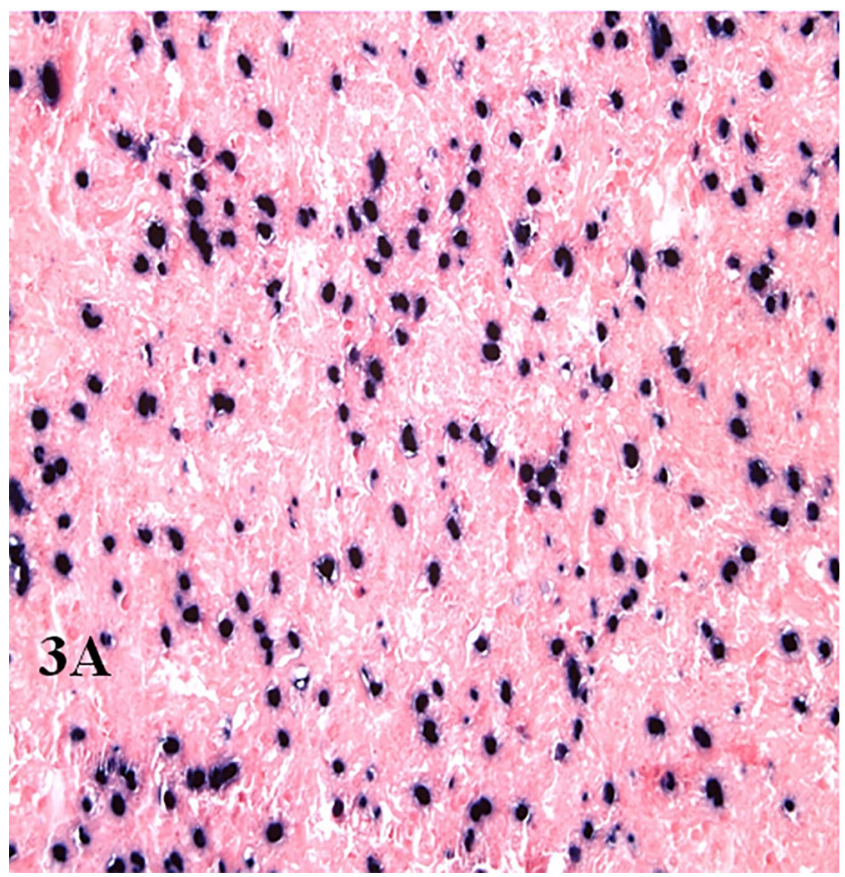

kératinisant selon OMS 2005 est à son tour scindée en carcinome différencié non kératinisant (ancien sous type II) et en carcinome indifférencié dit « undifferenciated carcinoma of nasopharyngeal type » (UNCT : ex type III), qui est l'objet de ce travail. Il s'agit d'une entité histologique rare, ayant une répartition géographique disparate à travers le monde, superposable à celle des cancers du nasopharynx en général, avec des zones de forte incidence, de moyenne incidence et de faible incidence [10]. Le Sénégal n'est pas répertorié comme une zone de forte incidence et aucune étude n'a été à notre connaissance effectuée sur cette entité en particulier. À noter qu'il s'agit du type histologique prédominant dans les régions fortement endémiques où il peut représenter jusqu'à $90 \%$ de l'ensemble des carcinomes du nasopharynx [3].

Sur le plan épidémiologique, notre travail a concerné une courte série mais sur une longue période d'étude, ce qui traduit bien le caractère non endémique de ce type de cancer au Sénégal. L'âge moyen dans notre série est de 28,37 ans, donc relativement jeune par rapport à l'âge moyen en zone endémique où il est souvent compris entre la cinquième et la sixième décade [13]. Dans certains pays à risque, au Maroc par exemple, il y une distribution bimodale en termes d'âge de survenu des carcinomes du nasopharynx ; entre 25 et 30 ans d'une part et après 50 ans d'autre part $[6,8]$. L'âge moyen observé dans notre étude est plutôt superposable au premier intervalle d'âge observé dans les pays sus mentionnés (Maroc, etc.), c'est-à-dire celui compris entre 25 et 30 ans. Cette différence entre l'âge moyen dans notre série

Fig 3 Marquage nucléaire des cellules tumorales infectées par EBV en Hybridation in situ avec la sonde EBER / Immunolabelling of tumoral cells infected by EBV using EBER ISH probe 3A : marquage nucléaire diffus et modéré des cellules tumorales (Score : 2) ; 3B : marquage diffus et intense des cellules tumorales (Score : 3 ). 
par rapport à celui retrouvé dans les zones à risque élevé peut s'expliquer par le fait que le contact avec EBV se ferait essentiellement dans l'enfance dans les zones non endémiques, d'où la survenue un peu précoce du cancer ; alors qu'en zone à risque élevé il y a beaucoup de nouvelles infections à EBVy compris chez les adultes et donc l'âge moyen a tendance à avoir une distribution plus étendue. Nous avons par ailleurs observé dans notre série une nette prédominance masculine avec un sexe ratio de 8/3. Dans la littérature, il est aussi rapporté pour les UCNT comme pour les carcinomes nasopharyngés en général, une prédominance masculine, avec un rapport homme/femme de 2,5 ou 3 [13].

Sur le plan anatomo-pathologique, il convient de rappeler qu'une atteinte ganglionnaire est souvent présente au diagnostic et est souvent bilatérale. Cette atteinte ganglionnaire peut constituer l'élément révélateur de ce type de cancer. Dans notre série, une atteinte ganglionnaire cervicale était présente dans 11 cas sur 13 et elle était bilatérale dans 10 cas. Elle avait révélé le diagnostic dans 3 cas. À l'endoscopie, quand une lésion du cavum était notée, l'aspect macroscopique était celui souvent observé en pratique courante dans les carcinomes épidermoïdes en général indépendamment de la localisation, à savoir un caractère ulcérobourgeonnant des lésions. À l'histologie, il s'agit d'une lésion de diagnostic difficile par son caractère indifférencié et qui pose un problème de diagnostic différentiel avec certaines lésions lymphomateuses et certaines lésions sarcomateuses. Dans notre série, nous avons réalisé une étude immunohistochimique afin d'avoir la certitude diagnostique. Deux cas n'étaient finalement pas des carcinomes, ce qui montre l'intérêt de l'immunohistochimie dans ce type de lésion, car le pronostic et la prise en charge diffèrent par rapport aux sarcomes et aux lymphomes.

Dans l'étiopathogénie d'UCNT, la composante virale est essentielle, et il existe dans ce sens une liaison constante avec le virus d'Epstein-Barr avec une positivité de presque $100 \%$ dans la plupart des régions, et surtout celles qui sont endémiques $[1,2,14]$. Ceci a justifié dans notre série la recherche d'EBV en hybridation in situ avec la sonde EBER. La positivité EBER dans notre série était de $77 \%$. Il reste un pourcentage assez significatif de cas négatifs, de $23 \%$ $(\mathrm{N}=3)$. Les hypothèses suivantes peuvent être formulées et discutées par rapport à cette petite différence :

- la première hypothèse est que la qualité de conservation des prélèvements et les aléas de technique ont peut-être réduit les chances de mettre en évidence EBV en hybridation in situ;

- la seconde est que, en zone non endémique pour EBV, ces cas négatifs sont peut-être en rapport avec d'autres facteurs de risque.

Des études au Sénégal ont notamment montré le rôle par exemple du Papilloma Virus Humain (HPV) dans la sur- venue des cancers des voies aérodigestives supérieures [14]. Aussi, HPV est retrouvé dans le carcinome indifférencié du nasopharynx sur certaines catégories de population, américaine notamment [12]. Par ailleurs, Kano, au Japon [7], une zone pourtant non endémique pour HPV, a détecté HPV chez les patients porteurs de carcinomes du nasopharynx indépendamment du type histologique bien qu'ayant suggéré que ce virus n'aurait aucune influence sur la carcinogenèse de ces carcinomes dans la dite population.

Notre série nous pousse à nous demander si l'UCNT en zone supposée non endémique à EBV et au Sénégal en particulier, est nécessairement associé à Epstein-Barr virus. Il serait intéressant, au regard de ce que nous décrivons cidessus, de faire la recherche de HPV sur tous nos cas non EBV positifs.

\section{Conclusion}

En définitive, cette série dakaroise montre que le carcinome indifférencié du nasopharynx bien que rare au Sénégal y est tout de même diagnostiqué et qu'il faut y penser devant un carcinome non kératinisant OMS 2005 du nasopharynx. À Dakar, il touche en particulier des sujets de sexe masculin, relativement jeune. Son caractère indifférencié rend son diagnostic histologique difficile et montre l'intérêt de la disponibilité de l'immunohistochimie pour trancher entre ce diagnostic et les autres lésions tumorales malignes indifférenciées. Même dans cette zone supposée non endémique, le virus d'Epstein-Barr est en cause et sa recherche devrait être systématique. Ce virus n'est cependant toujours pas mis en évidence et peut légitimement nous pousser à s'interroger sur l'implication ou non d'autres facteurs de risque comme le Papilloma virus humain. Une série plus importante devrait nous donner des informations plus intéressantes bien que difficiles à obtenir dans une zone supposée non endémique.

Remerciements au professeur Henri Sevestre, chef de service du laboratoire d'anatomie et cytologie pathologiques du CHU d'Amiens.

Liens d'intérêt : les auteurs déclarent ne pas avoir de liens d'intérêt.

\section{Bibliographie}

1. Altun M, Fandi A, Dupuis O, et al (1995) Undifferentiated nasopharyngeal cancer (UCNT): current diagnostic and therapeutic aspects. Int J Radiat Oncol Biol Phys 32:859-77

2. Bar-Sela G, Kuten A, Minkov I, et al (2004) Prevalence and relevance of EBV latency in nasopharyngeal carcinoma in Israel. J Clin Pathol 57: 290-3 
3. Barnes L, Everson JW, Reichart P, Sidransky D (2005) World Health Organization Classifications of Tumours. Pathology \& Genetics. Head and Neck Tumours. IARC press L, editor, $435 \mathrm{p}$

4. Chang ET, Adami HO (2006) The enigmatic epidemiology of nasopharyngeal carcinoma. Cancer Epidemiol Biomarkers Prev $15: 1765-77$

5. Fleury B, Biston MC, Montbarbon X, Pommier P (2010) Cancers $\mathrm{du}$ cavum de l'adulte. Cancer Radiother 14:S23-33. doi: 10.1016/S1278-3218(10)70005

6. Jalbout M, Bel Hadj Jrad B, Bouaouina N, et al (2002) Autoantibodies to tubulin are specifically associated with the young age onset of the nasopharyngeal carcinoma. Int J Cancer 101:146-50

7. Kano M, Kondo S, Wakisaka N et al (2017) The influence of human papillomavirus on nasopharyngeal carcinoma in Japan. Auris Nasus Larynx 44:327-32. doi: 10.1016/j.anl.2016.07.015

8. Khabir A, Sellami A, Sakka M, et al (2001) Contrasted frequencies of 553 accumulation in the two age groups of North African nasopharyngeal carcinomas. Clin Cancer Research 6:3932-6

9. Nicholls JM, Agathanggelou A, Fung K, et al (1997) The association of squamous cell carcinomas of the nasopharynx with
Epstein-Barr virus shows geographical variation reminiscent of Burkitt's lymphoma. J pathol 183:164-8

10. Mimi CY, Yuan JM (2002) Epidemiology of nasopharyngeal carcinoma. Seminars in cancer biology-Elsevier. 126: 421-9. doi: 10.1016/S1044579X02000858

11. Parkin DM, Whelan SL, Ferlay J, et al (1997) Cancer Incidence in Five Continents. Vol. VII. International Agency for Research on Cancer, Lyon, $\mathrm{N}^{\circ} 143,1240 \mathrm{p}$

12. Punwaney R, Brandwein MS, Zhang DY, et al (1999) Human papillomavirus may be common within nasopharyngeal carcinoma of Caucasian Americans: investigation of Epstein-Barr virus and human papillomavirus in eastern and western nasopharyngeal carcinoma using ligation-dependent polymerase chain reaction. Head Neck 21:21-9

13. Spano JP, Busson P, Atlan D, et al (2003) Nasopharyngeal carcinomas: an update. Eur J Cancer 39: 2121-35

14. Woto-Gaye G, M'Farrej MK1, Doh K, et al (2016) Les papillomavirus humains: un autre facteur de risque des carcinomes des voies aérodigestives supérieures. Bull Soc Pathol Exot 109:160-4. doi: 10.1007/s13149-016-0500-7 [http://www.pathexo.fr/documents/ articles-bul1/2016_109_160-164.pdf] 